\title{
Le laboratoire du Commissariat à l'énergie atomique
}

\author{
G. PORTAL
}

La dosimétrie individuelle passive constitue un élément essentiel de la surveillance du personnel travaillant dans un milieu soumis au risque d'irradiation par des rayonnements ionisants. Elle est assurée par un détecteur de rayonnement, porté à la poitrine ou à l'emplacement soumis au risque (poignet, doigt). Elle est parfois accompagnée d'une dosimétrie active à lecture immédiate (stylo-électromètre, « Dosicard », etc.).

Le CEA, conscient de la priorité à réserver à la protection du personnel, en particulier, a fait un effort remarquable pour en développer les techniques qui devaient s'adapter, dès la fin des années 1950, à une diversification et une multiplication rapide des risques, dues à l'accélération des programmes nucléaires. Le Laboratoire du CEA assurait d'une part la surveillance dosimétrique des personnels du CEA et associés, mais aussi la veille scientifique de toutes les recherches sur les techniques de dosimétrie passive et active.

En matière de dosimétrie individuelle, presque tout était à créer.

Ayant été appelé à initier et animer de 1959 à 1992 la plus grande partie de cet effort, je retrace dans cet historique les recherches et les développements réalisés au Centre d'études de Fontenay-aux-Roses qui, tout au moins au début, regroupait la surveillance du personnel de la presque totalité du CEA et dont la mission était de mettre au point la dosimétrie externe des rayonnements ionisants et d'en diffuser les techniques au niveau national. J'ai fait appel pour cela à mes propres archives (notamment à mon dernier rapport DPH/2000-1(IPSN, 2000)). J'ai également bénéficié de l'appui de mes principaux collègues et amis qui ont bien voulu m' aider à rassembler tous nos souvenirs pour faire vivre ce document: Anne-Marie Chapuis, Yvonne Herbaut, Jean Barthe, Francis Bermann, Jean Louis Chartier, Roger Medioni et Maurice Petel.

Sont mentionnés dans ce document les noms de mes principaux collaborateurs qui ont participé, au fil de ces 35 années, à cette grande aventure « collective ». Y sont également cités divers acteurs français et étrangers extérieurs à notre service, 
afin d'honorer tous ceux qui ont participé à cette immense tâche et sensibiliser le lecteur à l'importance de la coordination des recherches en France et à l'étranger.

L'aspect technique de ces travaux n'est relaté ici que d'une façon très succincte, car rappelé dans la première partie de ce numéro.

L'attention y est avant tout focalisée sur la surveillance dosimétrique des photons et des électrons par émulsion photographique, seule technique admise par la réglementation depuis l'arrêté du 19 avril 1968 et cela jusqu'en 1999. On donne des informations sur les résultats des recherches qui ont abouti à la création du dosimètre individuel passif ainsi que sur les activités liées à ces recherches :

- création d'un ensemble de faisceaux de rayonnements de référence nécessaires pour l'étude des caractéristiques et l'étalonnage des détecteurs ;

- participation active à la préparation de multiples normes, indispensables pour assurer une garantie internationale au système de surveillance mis en œuvre au Centre CEA de Fontenay-aux-Roses appelé pour simplifier FAR.

Les recherches basées sur les phénomènes de physique du solide y sont également rappelées; elles sont à la base des techniques de dosimétrie individuelle utilisées depuis longtemps dans le monde et en France depuis la levée tardive de l'exclusivité de l'utilisation du film.

Les recherches et développements concernant la dosimétrie individuelle des neutrons y sont relatés. Elles ont constitué un volet important des activités du service.

Enfin, sont évoquées les collaborations au niveau français et européen qui ont permis d'aborder un champ d'investigation aussi large et de bénéficier du concours des principaux partenaires dans un effort général de création d'une dosimétrie mondiale de qualité, essentielle pour la crédibilité du nucléaire.

\subsection{Le lancement des premières études}

Francis Duhamel, chef du Service de contrôle des rayonnements et de génie radioactif (SCRGR) et Georges Soudain, chef du Groupe de recherche dosimétrique de ce service, m'avaient recruté en février 1959 pour créer à Fontenay-aux-Roses un Laboratoire de recherche en dosimétrie externe et dès le mois d'avril de la même année, pour prendre également la direction du Laboratoire d'exploitation dosimétrique (LED), appelé alors « Photométrie », dont le chef d'atelier était Léon Faye.

La mission était :

- au laboratoire de recherche, de mettre au point la future dosimétrie des rayonnements externes ; 
- à la photométrie, de faire face dans l'immédiat à l'explosion du nombre de personnes surveillées et d'y mettre progressivement en service les nouveaux dosimètres qui devaient être conçus.

Le laboratoire initial de recherche, situé à FAR, est devenu au fil des années un service important, inclus dans le Département de protection technique, dirigé par Jacques Pradel. Le nom du laboratoire ayant été maintes fois modifié au cours des décennies (notamment : Section d'étude et d'instrumentation en dosimétrie (SEID), Service d'instrumentation en dosimétrie et en radioprotection (SIDR), Service de dosimétrie (S.DOS) pour devenir un service, nous l'appellerons ici, par commodité, le «SIDR » (Service d'instrumentation et de dosimétrie en radioprotection). Il incluait une annexe spécialisée dans la dosimétrie des neutrons, à Cadarache, et a totalisé jusqu'à plus de 70 personnes: médecins, physiciens, ingénieurs, techniciens et ouvriers auxquels s'ajoutaient des stagiaires et de nombreux doctorants.

\subsection{Le dosimètre photographique}

\subsubsection{L'origine du dosimètre basique CEA}

Le dosimètre utilisé jusqu'en 1959 à FAR, était constitué du film Kodak type «dentaire » combiné à des écrans très simples comportant, selon les postes de travail, un couple d'écrans «étain-cadmium », pour la surveillance auprès des sources radioactives et des réacteurs, ou « cuivre $0,2 \mathrm{~mm}$-cuivre $0,6 \mathrm{~mm}$ », pour les personnes travaillant auprès d'installations à rayons $\mathrm{X}$. Il était alors appelé «pince dosimétrique gamma ou rayons $\mathrm{X}$ » selon les cas.

Ces écrans avaient été conçus par le chef de laboratoire d'origine, Georges Soudain (bien avant mon arrivée au CEA) en collaboration avec Paul Bonet-Maury du CNRS. Il était bien adapté à la situation de la surveillance du personnel à la fin des années 1950 : simplicité, faible coût, surveillance des irradiations X, gamma et neutrons thermiques.

Le laboratoire d'exploitation dosimétrique a dû faire face en 1960-61 à un accroissement considérable du nombre de personnes surveillées (d'environ 7000 par mois à 40 000). Même le personnel administratif a reçu un dosimètre. Il a fallu rationaliser précipitamment les opérations qui étaient jusqu'alors traitées sur une base semi-artisanale.

Ces écrans ont été adoptés par le SCPRI. Au début des années 1960, nous assurions sa dosimétrie. En 1964, alors que nous abandonnions les dosimètres basiques à FAR pour les remplacer par le nouveau dosimètre appelé PS1 que nous 
venions de concevoir, nous avons donné au Professeur Jean Pierre Moroni du SCPRI (qui avait bénéficié d'une formation rapide dans notre laboratoire), une grande partie de nos pinces porte-écrans ainsi que la machine à développer les films que nous n'utilisions plus. Les écrans utilisés par la suite au SCPRI (tout comme au LCIE) ont évolué par adjonction au cas par cas d'un carré de plomb et/ou de cadmium, comme le mentionne Alain Biau au chapitre 7. Il est certain qu'il était bien adapté aux besoins spécifiques de cet organisme.

\subsubsection{Pourquoi a-t-il fallu créer au CEA un nouveau dosimètre?}

Le CEA se développait rapidement, le risque d'accident de criticité devenait un souci majeur, les sources de rayonnement potentielles se multipliaient et se diversifiaient depuis les plus faibles jusqu'aux plus fortes énergies et le nombre de personnes à surveiller explosait. Il avait été notamment décidé d'attribuer un dosimètre à tout le monde, même à ceux qui n'avaient pas d'activité à risque.

Après une large enquête longue et laborieuse, entreprise auprès de tous les centres du CEA tel qu'il existait au début des années 1960 et incluant la COGEMA d'alors, les mines d'uranium en France et en Afrique et les clients associés au groupe CEA, nous sommes arrivés aux conclusions suivantes.

Il fallait concevoir un dosimètre répondant aux conditions définies ci-après :

1/ modèle unique pour l'ensemble des travailleurs ;

2/ capable de mesurer les irradiations gamma et bêta dans une large :

- gamme de dose ; de 20 millirads à 800 rads, grandeurs utilisées à l'époque (200 micrograys à 8 grays) permettant des mesures en cas d'accident grave ;

- plage d'énergie : de $10 \mathrm{keV}$ à plusieurs $\mathrm{MeV}$, permettant ainsi d'effectuer des évaluations dans tous les cas.

$3 /$ permettant d'obtenir une précision meilleure que $20 \%$, toutes erreurs confondues ;

4/ capable éventuellement de donner des informations complémentaires sur l'énergie du rayonnement et son angle d'incidence pour des enquêtes éventuelles ; $5 /$ pouvant être entièrement traité par des machines automatiques ;

6/ comportant des détecteurs complémentaires pour la détection des irradiations par des neutrons et couvrant le risque de criticité ;

7/ emballé obligatoirement dans un emballage étanche et soudé pour le mettre à l'abri de la contamination (figure 3a). Cette dernière spécification était très contraignante, nous interdisant de laisser le boîtier à l'utilisateur pour ne lui expédier que le film, comme pouvait le faire le SCPRI ;

8/ disposant d'un témoin de bon développement. 


\subsubsection{Le film Chassende-Baroz}

Partant du dosimètre mis au point par le général Norbert Chassende-Baroz pour les armées et avec ses conseils, nous avons étudié avec la société Kodak la possibilité d'ajouter une émulsion complémentaire pour couvrir la gamme de dose de 20 millirads à 800 rads (actuellement 200 micrograys à 8 grays). Nous en avons entièrement financé la chaine de fabrication. Ce film-dosimètre a été mis en service en 1963. Il est décrit par Alain Biau au chapitre 3. Il a été utilisé jusqu'en 1993, date à laquelle le nouveau responsable du laboratoire d'exploitation dosimétrique, Yvon Magri, a changé le film et a totalement renouvelé la chaîne d'exploitation.

\subsubsection{Le boitier PS1 à sept plages de lecture}

Sa conception a demandé trois années d'efforts importants : enquête des besoins sur l'ensemble du groupe CEA, étude théorique et calcul des écrans pour couvrir la gamme d'énergie spécifiée, conception des installations pour en analyser et vérifier les caractéristiques, mise au point des méthodes d'interprétation des doses, validation des performances, essais en petite série, avant-projet de chaîne automatique de traitement, etc. Il a été breveté, et présenté au Symposium de l'Agence européenne pour l'énergie nucléaire « Personal dosimetry techniques for external radiations » à Madrid en 1963 puis mis en service progressivement en 1964 (figure 8.1).

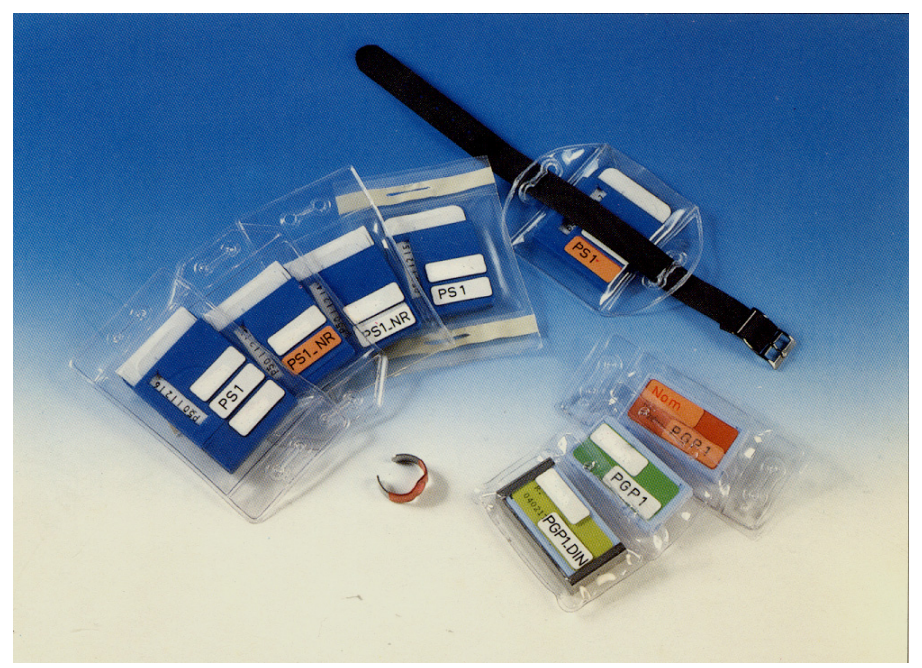

Figure 8.1 - Panoplie des divers dosimètres photographiques et thermoluminescents.

Par la suite, ont été ajoutés à ce boîtier divers detecteurs pour assurer la dosimétrie en cas d'accident de criticité constituant le PS1-C (figure 8.2). 

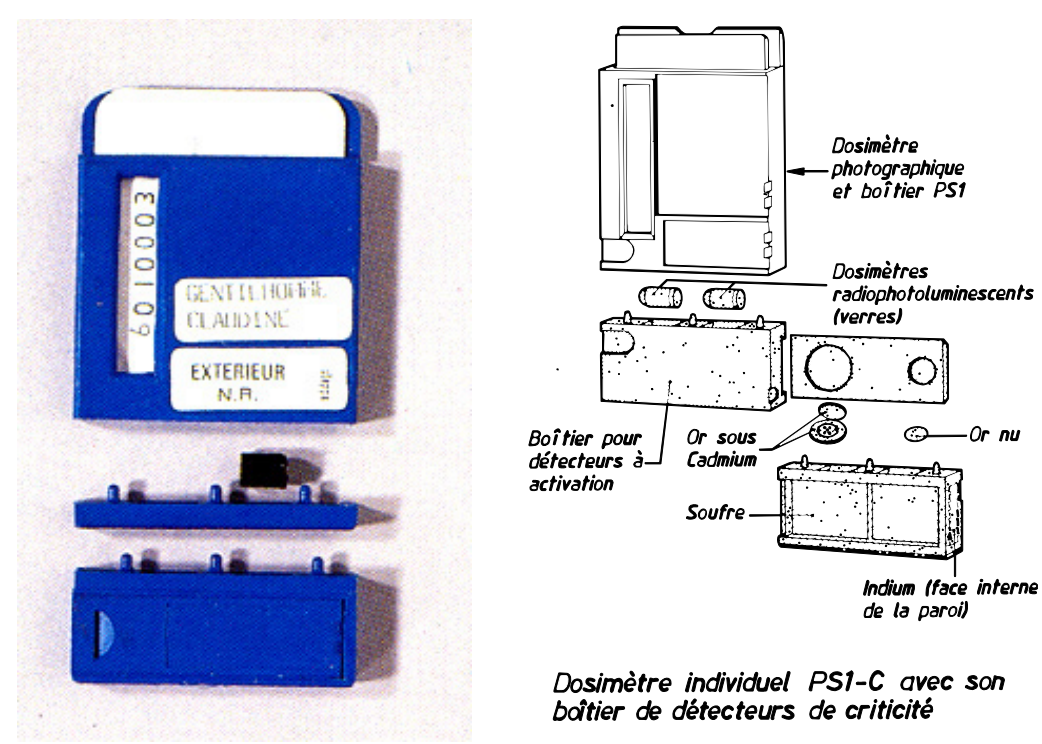

Figure 8.2 - Dosimètre individuel PS1-C avec son boitier de détecteur de criticité.

Une méthode de calcul simple, dite «empirique», n'exigeant pas d'interprétation humaine systématique, s'est substituée un peu plus tard à l'utilisation de corrections énergétiques par comparaison avec des étalons, simplifiant ainsi la détermination des irradiations et assurant une meilleure précision. Il suffisait d'insérer les résultats des lectures de doses apparentes sous chaque plage de lecture dans une formule de calcul dans laquelle étaient introduits des coefficients déterminés empiriquement. Par la suite, Francis Bermann, qui nous avait rejoints pour nous seconder et diriger le Laboratoire d'exploitation dosimétrique, a mis au point une méthode de calcul qui permettait de déterminer le spectre d'irradiation, élément important en cas d'enquête sur l'origine d'une irradiation.

Il est à noter que nous avions participé à l'installation d'un laboratoire de photométrie à Bruyères-le-Châtel (Bâtiment III du CEN/FAR) qui traitait l'ensemble des dosimètres de la Direction des applications militaires (DAM) ; plus tard, le traitement de ces dosimètres a été réintégré à celui de FAR. Des ingénieurs du LCIE, de la COGEMA (Marcoule et La Hague) et d'EDF ont fait des stages de formation au laboratoire et se sont inspirés de nos études pour concevoir des dosimètres dérivés du PS1, spécialement adaptés à leurs besoins spécifiques. Jean Pierre Moroni a toujours suivi nos travaux de près. 


\subsubsection{La chaîne automatique de traitements des films}

C'est Paul Marchal qui avait été chargé de concevoir la chaîne automatique de traitement. Elle a été mise en service en 1966. Elle effectuait toutes les opérations automatiquement depuis l'extraction du film de son boitier, l'ouverture de l'emballage, le développement, l'identification et le classement. Un seul bémol dans cette mécanisation: compte tenu de la facilité de sélection visuelle des dosimètres irradiés sur le « film » Chassende-Baroz et de leur très petit nombre (1 à $2 \%$ du total), la partie photométrique de la chaîne est restée à l'état de prototype. Il s'est avéré plus simple de continuer la sélection visuelle des dosimètres ayant reçu des doses supérieures à 20 millirads (200 micrograys) et d'évaluer les irradiations significatives à l'aide d'un photomètre classique pour les doses supérieures à 50 millirads (0,5 milligrays) qui représentaient moins de $1 \%$ des dosimètres). Le prototype que nous avions construit n'a pas eu de successeur industriel : le gain de temps eût été trop faible. Cette chaîne de traitement a été progressivement modifiée et simplifiée au fil des années par Francis Bermann, qui assurait la direction du laboratoire d'exploitation dosimétrique et qui devait tenir compte de la réduction du nombre de films dosimétriques traités et de l'usure de l'installation.

\subsubsection{La précision des mesures}

Un programme strict de «surveillance qualité » avait été mis rapidement en place et confié à Roger Marsault. Il mettait en œuvre de nombreux dosimètres « espions » irradiés par le LCIE (Laboratoire central des industries électriques, agréé par le Bureau national de métrologie, BNM) et mélangés à nos propres dosimètres et à nos étalons. Il nous a permis de maîtriser la qualité d'une façon remarquable. Cela est apparu d'une façon éclatante quand Euratom (la DG5 puis la DG11, avec Hans Eriskat et Heinz Seguin) a organisé des intercomparaisons entre les divers laboratoires européens.

Pour les rayons $\mathrm{X}$ et les rayons gamma, les résultats, avec une précision supérieure à $5 \%$ (dans les conditions strictes d'étalonnage), nous ont propulsés en tête du peloton et nous ont rassurés sur la qualité de nos étalons et de nos mesures. Les laboratoires de référence étaient le Physikalisch Technisch Bundesanstaldt (PTB) de Brunswick en Allemagne (sous la direction du Professeur Siegfried Wagner) et le National Physical Laboratory (NPL) en Angleterre (avec Alan Jennings); nous avions ainsi à notre disposition des références indépendantes des nôtres, qui se trouvaient confirmées internationalement.

Les résultats ont été tout aussi spectaculaires pour les neutrons thermiques ; nous avons nous mêmes été étonnés que nos résultats soient supérieurs à $10 \%$ de ceux 
du faisceau de neutrons thermiques du PTB, alors que les autres laboratoires commettaient des erreurs avoisinant les $100 \%$. Nous le devions à l'excellence de l'étalonnage de la colonne de neutrons thermiques de la pile Zoé, étalonnage effectué par les techniciens du réacteur.

Nous avons bénéficié de ces intercomparaisons pendant de nombreuses années, jusqu'à ce que le SCPRI nous demande de ne plus y participer car c'était son rôle et non celui de l'Euratom, de nous contrôler. À la place de ces intercomparaisons, nous avons fourni au SCPRI des dosimètres qui étaient portés en double avec les siens. Les résultats de ces contrôles ont été parfois incohérents. Francis Bermann ou moi-même avons rencontré plusieurs fois le Professeur Jean-Pierre Moroni sans pouvoir toujours déterminer l'origine de ces différences. Heureusement que notre système de « contrôle qualité », qu'il connaissait parfaitement et qui a été maintenu au moins jusqu'à mon départ à la retraite, nous mettait à l'abri de tout soupçon.

Il était regrettable que l'on ne puisse plus participer aux intercomparaisons internationales ; la comparaison est toujours une source de progrès. En fait et non officiellement, nous participions à la plupart d'entre-elles, car nous étions devenus un centre de référence comme le PTB et le NPL pour ces intercomparaisons et une partie des irradiations était effectuée chez nous. Nous pouvions donc comparer nos résultats à ceux des autres laboratoires ; simplement nos résultats n'étaient plus publiés.

Après une informatisation complète, réalisée par Fahimé Robiolle (aidée par une société extérieure, la GIXI), de la gestion des appareils de mesure et de la transmission des résultats à tous les clients, aux SPR, aux services médicaux et au SCPRI, le dosimètre PS1 semble avoir donné satisfaction puisqu'il a été utilisé pendant 43 ans, jusqu'en 2007.

\subsubsection{Les installations de référence "photons »}

Pour l'étude « fine » des caractéristiques énergétiques des dosimètres et pour leur étalonnage, il fallait disposer de rayonnements de référence bien caractérisés et reliés aux références nationales ou internationales. C'est un effort considérable qui a été entrepris dès le début et qui s'est prolongé même après mon départ, sous la direction de Jean-Louis Chartier.

\section{Rayonnement gamma et rayons $X$}

Nous nous sommes équipés d'une installation «panoramique » d'irradiation au cobalt conçue dans le service de Jean Vertut (voir photographie, figure 8.3) Ensuite, et sous la férule de Jean-Louis Chartier aidé de Christian Itié, nous avons mis en 
service un ensemble de générateurs de rayons $\mathrm{X}$ capables de fournir, non seulement les spectres plurichromatiques normalisés produits entre $60 \mathrm{kV}$ et $300 \mathrm{kV}$, mais également les faisceaux de rayons $\mathrm{X}$ monochromatiques de $8 \mathrm{keV}$ à $100 \mathrm{keV}$, essentiels pour l'étude de la réponse énergétique et angulaire des dosimètres. Il est à noter que parallèlement à cette étude, Yvonne Herbaut a créé à Grenoble, sous la direction d'Henri de Choudens, des installations similaires. Nous avons également créé, en collaboration avec le LCIE, des faisceaux « canalisés » de ${ }^{60} \mathrm{Co}$ et de ${ }^{137} \mathrm{Cs}$, conformes aux spécifications du Laboratoire de métrologie des rayonnements ionisants (LMRI).

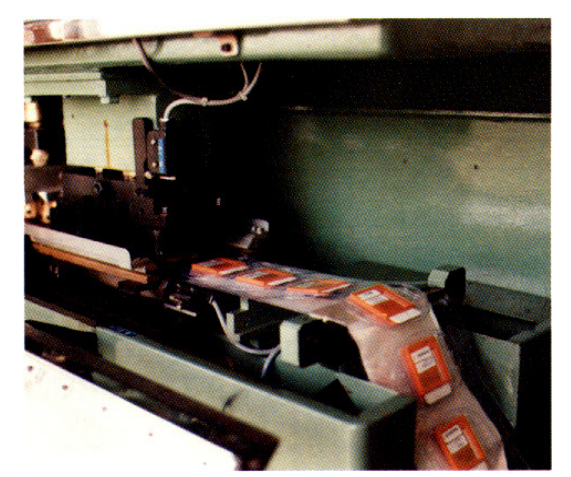

(a)

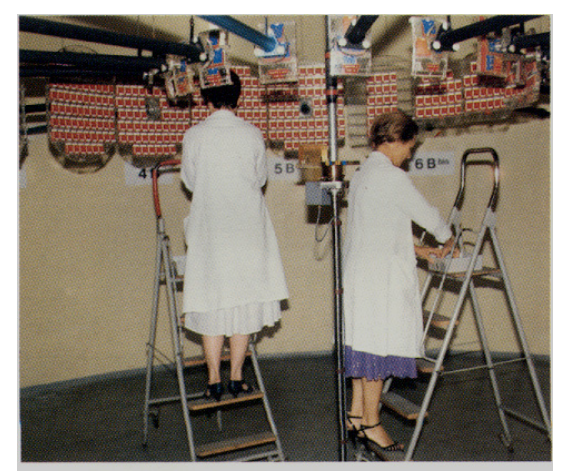

(b)

Figure 8.3 - Conditionneuse (a) et salle panoramique d'irradiation (b).

\section{Photons de haute énergie}

Francis Bermann, avec l'aide de Gilbert Troesch et de Claude Lasseur (du SPR de FAR), a créé auprès du réacteur Triton une installation de production de photons monochromatiques de 6 et $9 \mathrm{MeV}$ obtenus par interaction de neutrons thermiques sur des cibles de titane et de nickel. Wolfgang Alberts, qui avait suivi ces travaux de près, a installé par la suite le même dispositif au PTB de Brunswick (Allemagne). Celle de FAR fut utilisée en 1975 par la Commission des communautés européennes (CCE) pour des intercomparaisons internationales. Après l'arrêt de ce réacteur, il l'a transférée auprès du réacteur ISIS qui fut lui-même arrêté un peu plus tard.

\section{Rayonnement bêta}

Jean Louis Chartier a développé, avec l'aide de Dominique Cutarella et en liaison étroite avec le PTB de Brunswick (Jürgen Bohm) et le SPR de Grenoble (Yvonne Herbaut), des sources de référence pour le rayonnement bêta. Ont participé à ces 
G. PORTAL

études Gérard Audoin, René Chuiton et Jean-Claude Chapuis. Nos travaux ont abouti à l'élaboration d'une série de normes dont la dernière est la norme ISO 6980 et à l'organisation d'une conférence internationale à Saclay en 1985.

\section{Sources de rayonnement à impulsions}

À cela il faut ajouter qu'auprès des accélérateurs «Euphrosine » et « Tally » de Valduc, dans le cadre d'un groupe de travail inter SPR (le GTN1), Francis Bermann avec Philippe Blanchard et Michel Hulot ont testé la réponse des détecteurs aux sources « à impulsions » avec une durée du « flash » d'environ 100 nanosecondes. Les résultats ont montré que, si le film Chassende-Baroz résistait bien à l'effet de flash, il n'en était pas de même pour le film Dupont ; il sous-estimait légèrement les doses.

\section{Criticité}

Il faut noter également les expériences de dosimétrie de criticité effectuées, d'abord auprès du réacteur « $\mathrm{CRAC}$ » par Paul Marchal, puis auprès du réacteur « Silène » de Valduc par Roger Medioni, expériences qui nous ont amenés à organiser, avec les ingénieurs du centre, plusieurs intercomparaisons européennes auprès de cette installation unique au monde (en 1970 sous l'égide de la CCE et, en 1993, sous l'égide de l'AIEA et du DOE) ainsi qu'un congrès international sur la dosimétrie de criticité en juin 1993.

Bien entendu, l'étalonnage de toutes ces installations était relié selon les cas aux références du LMRI (pour les sources radioactives de photons uniquement) du PTB ou du NPL pour tous les autres rayonnements.

\subsection{Le dosimètre à thermoluminescence}

Dès le lancement du dosimètre boitier PS1, nous avions le sentiment que le film n'était pas éternel et qu'il fallait s'orienter pour le futur vers d'autres techniques. La dosimétrie par thermoluminescence commençait à pointer le bout de son nez à l'étranger. En effet, la dépendance énergétique de certains matériaux TLD était infiniment meilleure que celle des films. Le résultat n'exigeait pas d'effectuer les corrections importantes nécessaires pour le film et l'exploitation en était plus simple. Nous avons entrepris des recherches sur cette technique.

Le délégué à la protection au CEA, André Gauvenet, suivait de très près ces recherches et les finançait dans l'espoir de mettre en place un système de surveillance local, permettant d'obtenir des résultats rapides en cas de suspicion d'irradiation. Avec ses encouragements, nous avons commencé à nous intéresser 
à la fabrication d'un premier matériau, le fluorure de lithium (LiF). Celui-ci, fabriqué alors aux États-Unis par la société Harshaw, nécessitait une régénération systématique après utilisation. Nous avons découvert avec Yveline Bails et Philippe Blanchard, qu'il était possible d'améliorer la stabilité du matériau par adjonction de sodium qui réduisait la température de cristallisation. La régénération n'était alors effectuée que toutes les 20 mesures environ.

Par ailleurs, nous avons mis au point la technique du « palier de préchauffage » dans l'appareil de lecture, technique qui a été tout de suite adoptée par tous les constructeurs mondiaux de lecteurs. L'élément détecteur appelé PTL et le palier de préchauffage ont fait l'objet de brevets internationaux en 1968 et de nombreuses publications internationales. C'est alors que nous avons pu concevoir un dosimètre passif individuel, le PGP1 (breveté en 1973) comportant quatre éléments détecteurs, placés derrière des filtres (fenêtre, matière plastique, cuivre, plomb), permettant de donner, comme information complémentaire à la dose, "l'énergie moyenne du rayonnement ». Nous avons en même temps conçu un lecteur ultra rapide, le LTGV, (breveté la même année) capable de traiter d'une façon entièrement automatique huit dosimètres PGP1 à la minute. Les quatre pastilles thermoluminescentes étant «lues» simultanément, 32 mesures par minute étaient donc effectuées. Les résultats numériques étaient enregistrés sur support informatique. Nous avions envisagé de conserver également, pour une éventuelle «deuxième-lecture», le signal de thermoluminescence (la courbe d'émission du détecteur). Cela ne s'est pas avéré utile et nous en avons différé la mise en service malgré les énormes progrès de la capacité de mémoire informatique. L'électronique en a été réalisée par les électroniciens du laboratoire sous la direction de Raymond Prigent.

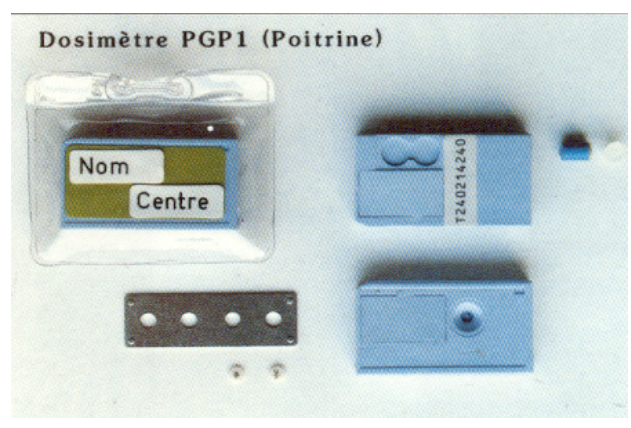

Figure 8.4 - Dosimètre PGP1 équipé de 2 pastilles $\mathrm{LiF}(\mathrm{g}+\mathrm{b})$ ou 4 pastilles pour déterminer l'énergie de rayonnement ou, pour le détecteur à neutron PGPI DIN, il y a un verre.

À ma connaissance, 37 ans après et malgré l'utilisation d'un chauffage laser par Jean Gasiot de l'université de Montpellier, il n'existe pas d'appareil automatique aussi rapide dans le monde. 
Notre lecteur a fonctionné de 1973 jusqu'en 2007 mais n'a pas pu être utilisé pour la surveillance du personnel directement affecté aux travaux sous rayonnement (DATR), comme cela était initialement l'objectif. En effet, entre temps, la réglementation française avait imposé l'utilisation exclusive du film photographique (arrêté du 19 avril 1968). Ce lecteur a été utilisé pour traiter les dosimètres du personnel non directement affecté aux travaux sous rayonnement (NDATR), pour les dosimètres neutrons à albédo (munis de pastilles de LiF 6 et LiF 7), pour les dosimètres complémentaires (surveillance au niveau du poignet et du doigt) et pour les dosimètres ultrasensibles au sulfate de calcium, mis au point par Serge Lorrain, utilisés pour les mesures d'environnement. Cet ensemble de dosimètres à thermoluminescence augmentait le nombre de résultats mensuels du laboratoire qui, dans les années 1990, n'était plus que de 30000 environ.

Les recherches concernant la dosimétrie par thermoluminescence n'ayant plus désormais la priorité au CEA, notre laboratoire a consacré une plus grande partie de son temps à la conception de dosimètres actifs.

Nous avons cependant participé à de nombreuses intercomparaisons européennes et initié la préparation de normes ISO sur la dosimétrie par thermoluminescence.

Ces recherches se sont également révélées utiles dans le cadre de la lutte contre le cancer car nous avons développé un lecteur de laboratoire (le LTV) et un lecteur automatique rapide (le PCL) utilisant le matériau PTL pour le suivi in vivo des irradiations des patients. Ces appareils ont été produits industriellement. Au sein de la Société française des physiciens médicaux (SFPM), elles nous ont amenés à épauler notre conseiller scientifique, le Professeur Daniel Blanc, pour organiser à Toulouse le premier congrès de cette association (voir la photo, figure 8.4).

Parallèlement, elles nous ont permis de participer aux programmes spatiaux européens, russes et américains ; il suffisait en effet d'un gramme de matière pour évaluer les irradiations des expériences embarquées (vols Apollo 16 et 17 sur la Lune, vols Rendez-vous Russo-Américain, Bioblock, Biostack, Exoblock).

Ces études nous ont également donné l'occasion d'organiser avec le Professeur Daniel Blanc, à Toulouse, la sixième conférence internationale sur la dosimétrie par l'état solide et une conférence sur la dosimétrie spatiale, à Strasbourg, sous l'égide du Conseil de l'Europe (Georges Massuet) en 1994.

\subsection{La dosimétrie par radiophotoluminescence}

Nous nous y étions intéressés au début comme détecteur complémentaire en cas d'accident. En relation avec Daniel Carpentier de la société CEC (Carbonisation entreprise céramique), Henri Francois, alors chef de la section dans laquelle le SIDR 
était inclus, Anne-Marie Chapuis et Anne Marie Grand Clément avaient développé un verre dosimétrique (breveté en 1962, 1965 et 1967) aux caractéristiques exceptionnelles pour l'époque. L'élaboration avec un barbotage d'oxygène lui conférait un faible bruit de fond; la présence de béryllium lui donnait une grande résistance à l'humidité et permettait de baisser son numéro atomique moyen et donc de diminuer l'hypersensibilité aux rayons X de faible énergie. Son seuil de détection était de 1 rad (10 milligrays) alors que celui de J.H. Schulman, de la NASA à Washington, était de 100 rads (1 gray). Nous l'avons adopté, après avoir modifié sa formule chimique et isotopique par inclusion de lithium, afin de lui donner la même réponse pour le même équivalent de dose de neutrons thermiques et de rayonnement gamma.

Nous avons ainsi crée le dosimètre PB1, mis au point avec Yveline Bails et industrialisé un lecteur de verre étudié par Raymond Prigent et Roger Chuiton. Ce détecteur complémentaire a été systématiquement inséré dans le logement du dosimètre PS1 (figure 8.2), prévu à cet effet en cas d'accident d'irradiation. Il permettait d'effectuer des mesures jusqu'à des doses très élevées et il était insensible à l'effet de flash (irradiation très brève). De plus, il permettait de faire des premières mesures sur place, en cas d'accident. Malgré des performances de sensibilité remarquables pour l'époque, il ne permettait pas d'effectuer la mesure des faibles doses. La question s'est posée d'abaisser ce seuil de détection. Il s'agissait davantage d'un sujet de recherche fondamentale car il n'était plus question de trouver une solution alternative à l'émulsion photographique.

Le sujet de recherche a alors été confié, sous contrat, au Professeur Daniel Blanc (conseiller scientifique du service). Le chercheur pressenti, Jean Barthe, a utilisé une méthode originale d'analyse du signal émis par le verre, à l'aide d'un laser à azote, qui permettait de séparer le bruit de fond de celui dû à l'irradiation. Cela lui permettait de détecter des doses extrêmement faibles. Il a présenté sa thèse en 1978 et nous a rejoints en 1981.

Cependant, l'absence dans le commerce d'un laser à azote performant, ne permettait pas alors d'utiliser cette méthode pour la dosimétrie courante. D'autre part, la mise au point de la dosimétrie par thermoluminescence était très avancée et l'exclusivité accordée à l'émulsion photographique ne nous encourageait pas à la développer en France. C'est cette invention qui a été reprise par les industriels japonais (Toshiba puis Chiyoda Tehnol Corporation) et qui est actuellement utilisée à l'IRSN.

\subsection{Pourquoi avoir imposé l'exclusivité du film argentique?}

De multiples arguments ont été avancés en faveur du film; aucun n'était suffisant à nos yeux pour le favoriser. Pourquoi l'avoir imposé en exclusivité alors que des techniques bien plus satisfaisantes se développaient rapidement dans le monde et 
avaient déjà atteint un stade de maturité tout à fait suffisant ? On ne saurait répondre à cette question, tout au moins sur le plan technique.

C'est une option qui bloquait définitivement toute innovation en France, la rétrogradant au niveau des pays les plus modestes en matière de surveillance du personnel alors que les plus grands pays nucléaires adoptaient ces techniques modernes. L'industrie française, qui avait pourtant le vent en poupe avec une avance non négligeable en matière de thermoluminescence à la suite de nos travaux, a stoppé ses investissements. En ce qui nous concerne, nous avons dû utiliser parallèlement deux systèmes différents selon que l'on traitait les DATR ou les NDATR.

Ce n'est qu'en 1999 que cette exclusivité a été levée et que nos successeurs ont pu utiliser d'autres techniques. Il aura donc fallu attendre longtemps pour que l'on adopte une méthode plus moderne. Que de temps perdu et quel gâchis pour l'industrie française ! On peut se consoler en constatant que la qualité de la surveillance du personnel n'a jamais faibli en France, ce qui était l'essentiel. On peut même affirmer, après avoir visité de nombreux laboratoires de dosimétrie individuelle dans le monde, qu'en France, le sujet était tout particulièrement pris au sérieux.

\subsection{La dosimétrie des neutrons}

Un effort tout aussi important que ce qui est décrit dans les paragraphes précédents lui a été consacré.

\subsubsection{Dosimètre NTA à émulsion nucléaire}

Ce fut d'abord le lancement en 1961 du dosimètre à émulsion nucléaire de type NTA, fabriqué par la société Kodak. Ce dosimètre, détectant les neutrons de 1 à $15 \mathrm{MeV}$, ne pouvait être conservé que sur une courte période (15 jours au maximum). L'analyse de l'origine de l'instabilité de l'image latente m'a permis de mettre au point une méthode de dessèchement de l'émulsion et de conditionnement sous azote dans un emballage étanche à l'humidité. Des dosimètres portés pendant un mois, en double, ont accusé une perte d'information de $50 \%$ pour ceux qui n'étaient pas protégés ; cela dépendait bien entendu des conditions d'humidité, de température et surtout de l'énergie des neutrons. Cette méthode, publiée à Madrid lors du symposium de 1963 évoqué plus haut, fut immédiatement adoptée par de nombreux laboratoires étrangers.

Il a fallu étudier la réponse énergétique de ce détecteur auprès de divers accélérateurs et l'équilibrer à l'aide d'un emballage adéquat. La « lecture » des traces de protons de recul étant effectuée au microscope, un effort de semiautomatisation a permis de diviser par cinq le temps nécessaire à l'évaluation des doses, il fallait ainsi trois minutes environ pour lire un dosimètre. Cependant, ce 
détecteur ne permettait pas la mesure des neutrons de faible énergie. Il fallait trouver des solutions complémentaires.

\subsubsection{Le dosimètre à albédo}

Le dosimètre NTA n'étant pas en mesure d'enregistrer les neutrons d'énergie inférieure à $1 \mathrm{MeV}$, il a fallu créer un détecteur complémentaire. C'est le dosimètre à albédo mettant en œuvre des pastilles thermoluminescentes sensibles aux neutrons thermiques diffusés par le corps du porteur soumis à un rayonnement complexe de neutrons.

Un boitier similaire au dosimètre à thermoluminescence PGP1 a été conçu ; on l'a appelé « PGP1-DIN », Les études de base avaient été effectuées au laboratoire annexe de celui de FAR, à Cadarache, par Michel Buxerolle, Jacques Kurdjian et Michel Mourgues. Le PGP1-DIN a été mis en service en 1981. Il était constitué de deux pastilles thermoluminescentes de ${ }^{6} \mathrm{LiF}$, sensibles aux neutrons thermiques et aux photons et deux de ${ }^{7} \mathrm{LiF}$, sensibles uniquement aux photons. Par différence, on évaluait la dose de neutrons thermiques.

Dans le cadre du risque d'accident de criticité, ce dosimètre devait être étalonné à chaque poste de travail et ses résultats devaient être reliés à ceux des détecteurs de site à activation SNAC placés à proximité. L'étude de ces derniers avait été initiée par Michel Bricka et poursuivie par Michel Buxerolle, Thadée Moraveck, Jacques Kurdjian et Michel Mourgues. Cette technique, impliquant un étalonnage à chaque poste de travail, n'était pas aussi satisfaisante qu'on l'aurait souhaité et diverses études avaient été lancées pour mettre au point un détecteur « actif » électronique : détecteur à sphères de Bonner (Michel Buxerolles), bisphères (Dineutron de Michel Mourgues), détecteur à émission exo-électronique (Maurice Petel), détecteur à Streamer (Michel Mourgues), détecteur à diode (Jean-Marc Bordy et Jean Barthe), compteur proportionnel multicellulaire équivalent tissu (Jean Barthe), détecteur de champ de rayonnement (Nguyen Van Dat a installé ses détecteurs à bord des satellites et des avions d'Air France), utilisation de la résonnance paramagnétique électronique (Francis Bermann, Jean Barthe, Vassiliky Kamenopoulou, Jean-Marc Bordy).

\subsubsection{La dosimétrie par détecteurs solides de traces (DST)}

Dès 1967, nous nous sommes intéressés à cette technique car, initialement soumise à une lecture au microscope des traces laissées dans le matériau plastique recouvert d'un convertisseur, elle s'est avérée rapidement automatisable après attaque électrochimique.

Les premières études sur ce matériau plastique avaient permis à Anne-Marie Chapuis de concevoir et breveter un dosimètre individuel pour la mesure de l'énergie alpha potentielle due aux descendants du radon. La réalisation et 
l'exploitation de ce dosimètre avaient ensuite été confiées à la COGEMA, puis à AREVA. Tous les mineurs des exploitations d'uranium en France et en Afrique en avaient été équipés.

À partir de 1977, Jean-Marc Bordy et Roger Medioni ont travaillé sur l'utilisation du CR39 et du CN85 pour la dosimétrie des neutrons. La reproductibilité de la fabrication du CR 89 laissait alors à désirer et la fabrication du CN 85 a été arrêtée par Kodak. Nous avons préféré en différer l'utilisation. Un peu plus tard, la technique est devenue accessible industriellement et utilisée couramment.

\subsubsection{Les installations de référence «neutrons»}

\section{Rayonnements poly-énergétiques}

Des installations de référence ont été créées dans l'annexe du service, au Centre d'études nucléaires de Cadarache, pour obtenir des faisceaux de neutrons de référence. Ce laboratoire était équipé de deux accélérateurs SAMES $(300 \mathrm{kV}$ et $150 \mathrm{kV}$ ), pouvant produire respectivement des neutrons de $3 \mathrm{MeV}$ (cible de deutérium) ou de $14 \mathrm{MeV}$ (cible de tritium) irradiant un convertisseur d'uranium 238 associé à une «chambre à diffusion » (Canel Plus). Celle-ci, imaginée par Michel Buxerolle sous la direction de Michel Bricka, était destinée à simuler le spectre de rayonnement que l'on rencontrait dans certaines situations réelles d'irradiation des travailleurs. Ont participé à ces travaux Jacques Kurkdjian, Martine Massoutie, Michel Mourgues et Jean-Louis Chartier. Elle a été présentée pour la première fois en 1992. Étant unique au monde, elle a été utilisée pour plusieurs intercomparaisons internationales organisées sous l'égide de la CCE et du DOE et a donné naissance à une norme (ISO 8529).

\section{Rayonnements mono-énergétiques}

Jean-Louis Chartier, avec l'aide de Christian Itie, Françoise Posny, Gérard Audoin, Jean Poitreau et Michel Sueur a également mis en place, auprès du Van de Graaf 5 MV du Centre d'études nucléaires de Bruyères-le-Châtel, une installation produisant des neutrons monochromatiques dans la gamme d'énergie de $140 \mathrm{keV}$ à $19 \mathrm{MeV}$ à partir des réactions $(\mathrm{p}, \mathrm{Li}),(\mathrm{p}, \mathrm{T}),(\mathrm{d}, \mathrm{d})$ et $(\mathrm{d}, \mathrm{T})$. Il s'agit d'un faisceau de neutrons dont l'énergie est déterminée par l'angle d'émission de ceux-ci par rapport à la direction des particules chargées incidentes sur la cible de lithium, de deutérium ou de tritium selon le cas.

\subsection{La normalisation}

Conscients de la nécessité de créer des normes internationales pour garantir la validité des mesures, nous avons participé à l'élaboration des projets de normes et, 
progressivement, nous en sommes devenus les initiateurs. L'AFNOR a créé un premier groupe de travail pour la préparation d'un projet sur le dosimètre photographique, initialement présidé par Robert Hignette d'EDF. J'ai ensuite été amené à prendre sa succession. Deux normes ont alors été éditées (ISO 1757 et sa version révisée). De la même façon, j'ai initié à l'AFNOR, parmi d'autres projets, tous ceux concernant la production de rayonnements de référence et ceux voués à la dosimétrie par thermoluminescence. Ayant été nommé président du SC2 (Sous-comité 2 de l'ISO chargé des normes concernant la radioprotection et agissant au sein du TC 85 (normes sur le nucléaire)), j'ai pu faire adopter toutes ces normes au niveau international (ISO 4037, ISO 6980, ISO 8529, ISO 10647, ISO 12789 et bien d' autres).

Un point particulier doit être mis en exergue, c'est notre rôle dans la création des Grandeurs opérationnelles. La CIPR avait défini une grandeur, « l'Indice de dose », que nous ne pouvions pas utiliser en radioprotection car elle n'était pas additive. J'avais fait pression en 1979 à la fois sur la CIPR et sur l'ICRU, avec l'aide de Nicole West de l'AFNOR, alors présidente du SC2, pour réexaminer ce problème. En effet, cette anomalie apparaissait systématiquement dans la préparation de tous nos projets de norme. Après une longue période de scepticisme, les discussions ont commencé mais elles ont été très laborieuses. Pendant ce temps, suivant cette initiative et dans le cadre de la DG12 de la CCE, un groupe de travail a préparé un projet de « Nouvelles grandeurs opérationnelles, qui a été finalisé lors de notre réunion à Cadarache et édité en 1983 sous le nom de «New Operational Quantities » (EUR 8346). De nombreux laboratoires européens et américains participèrent aux travaux.

Ce projet fut finalement adopté par la CIPR qui le publia. Ce n'est qu'un peu plus tard que l'ICRU, alors présidé par un Français, le Professeur André Allisy, prit conscience de la nécessité de suivre le mouvement et confia, d'abord à T.E. Burling de Londres et ensuite à moi-même, la présidence du comité chargé de préparer des recommandations. Ont alors été publiées : en 1985 la recommandation ICRU 39, en 1988 l'ICRU 43, en 1992 l'ICRU 47, puis dans la lancée et après mon départ, en 1993, l'ICRU 51, puis l'ICRU 57/CIPR Publication $\mathrm{n}^{\circ} 74$. Ces grandeurs opérationnelles ont été ainsi mises en place définitivement. Il faut également signaler l'ICRU 66 (en 2001) consacrée à la mise en œuvre des grandeurs opérationnelles pour les neutrons.

Toutes ces actions, essentielles à nos yeux, mettaient en évidence les recherches effectuées à Fontenay-aux-Roses. C'était un peu la consécration de notre laboratoire qui était souvent l'initiateur de bien des innovations et était alors reconnu au niveau international.

\subsection{Les collaborations en France et à l'étranger}

Nous avions conscience, dès le départ, que la tâche était immense et que les effectifs de notre laboratoire (pourtant important avec environ 50 personnes impliquées dans 
les recherches) n'étaient pas à même de faire face à tout le programme de recherches que nous voulions mener à terme. Fort heureusement, dès les premières années, nous avons bénéficié des conseils de Daniel Blanc, notre conseiller scientifique, qui suivait en permanence et dans le détail toutes les phases de nos travaux et nous encourageait à multiplier les contacts aussi bien au niveau français qu'européen.

En France, diverses universités ont été appelées à collaborer avec nous (Toulouse, Limoges, Nice, Saint-Étienne, Paris VI) ainsi que le CNRS (Orsay et Strasbourg) pour nous épauler dans la partie fondamentale de nos recherches. Daniel Blanc et moi-même avions décidé de créer une association, les Laboratoires associés en radiologie et en dosimétrie (LARD). Celle-ci nous a permis de démultiplier et coordonner nos recherches ; plus de 20 ans après, cette association est toujours active sous la présidence de Libor Makovicka.

Il faut ajouter à cela les contacts étroits avec les services de radioprotection de chaque centre du CEA, avec les armées et avec EDF. Notre chef de département, Jacques Pradel, avait créé des groupes de travail (GTN) sur chaque thème de recherche. Ce sont eux qui nous contraignaient à « garder les pieds sur terre ».

Enfin, le soutien que nous avons apporté aux radiophysiciens des hôpitaux (Jean Chavaudra de Villejuif, Ginette Marinello de Créteil et bien d'autres qui, pour la plupart, avaient été mes élèves de DEA à l'Université Paul Sabatier de Toulouse) nous ont sensibilisés à l'importance de la reproductibilité des mesures en thermoluminescence, avec un objectif inférieur au pour cent.

\section{Au niveau européen}

C'est d'abord par l'intermédiaire de la Commission de la Communauté européenne que les liens ont été noués :

- avec l'Allemagne (Pr. Siegfried Wagner et Pr. Gunter Dietze du PTB de Brunswick et Pr. Reinhard Nink du PTB de Berlin, Pr. D.Wachsmann et Pr. Albrecht Kellerer de la GSF à Munich, Pr. Arthur.Scharmann de l'Institut Justus Liebig à Giessen) ;

- avec le Royaume-Uni (Alan Jennings du NPL);

- avec l'Italie (Pr. Gilgerto Busuoli de Bologne) ;

- avec la Tchécoslovaquie (Pr. Frantisek Spurny de l'Institut de Dosimétrie de Prague) ;

- avec l'Espagne (Pr. Francisco Fernandez de l'université de Barcelone).

Finalement, une association européenne a été créée, l'EURADOS, qui réunissait tous ces laboratoires et dont j'étais co-fondateur. En tant que conseiller scientifique du CEA et du CNRS, j' ai organisé en septembre 1994 une assemblée générale de cette association, au CNRS de Strasbourg. 


\section{Au niveau international}

Nous avions surtout des contacts avec les chercheurs du NBS à Washington (Margaret Ehrlich, Bob Schwarz et J.S. Cheka ) et du DOE (John Horan et Jane Cusimano de Hanford). C'est cet échange permanent d'informations, de stimulation concurrentielle, d'entraide et de confrontation des résultats qui nous ont dynamisés dans bien des secteurs d'activité. Nous étions jugés par nos pairs ; nul n'a jamais douté du sérieux de la dosimétrie en radioprotection en France.

Que tous les anciens du SIDR sachent combien je leur suis reconnaissant de m'avoir suivi pendant si longtemps ; que ceux qui m'ont aidé dans la préparation de ce document soient remerciés pour leur contribution.

Je dédie cet historique à Daniel Blanc qui nous a soutenus pendant presque toute la période couverte par ce document. Si nous avons correctement rempli notre mission de chercheurs, c'est à lui que nous le devons, il y veillait en permanence lors de toutes nos manifestations (figure 8.5).

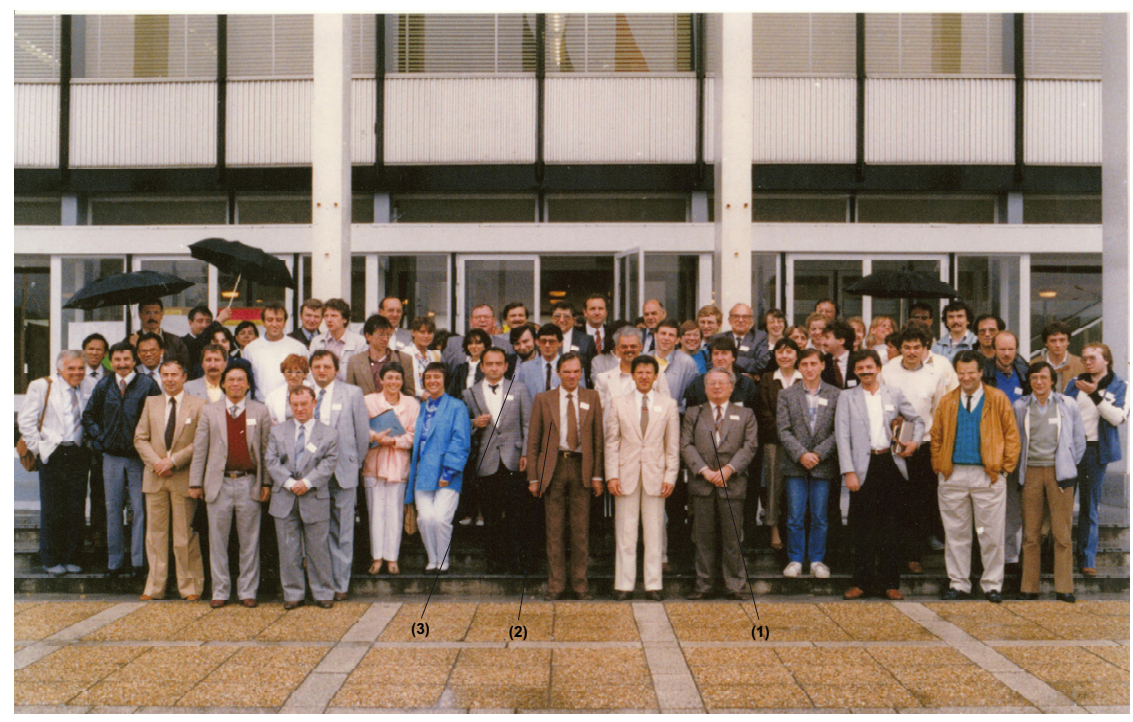

Figure 8.5 - Congrès de la Société française des physiciens d'hôpital organisé en 1986 à Toulouse par le Professeur Daniel Blanc (1) avec Gui Portal (2). Figure également Alain Biau, coordinateur de cet ouvrage (3).

\section{RÉFÉRENCE}

IPSN (2000) Institut de protection et de sûreté nucléaire, Rapport DPHD 2000-1, 2000. 